\title{
Single prolonged stress induces changes in the expression of mineralocorticoid receptor in the medial prefrontal cortex in a rat model of post-traumatic stress disorder
}

\author{
JING-HUA ZHANG ${ }^{1-3}$, FANG HAN ${ }^{1}$ and YU-XIU SHI ${ }^{1}$ \\ ${ }^{1}$ Department of Histology and Embryology, Basic Medical Sciences College; ${ }^{2}$ Institute of Pathology and Pathophysiology, \\ China Medical University, Shenyang, Liaoning $110001 ;{ }^{3}$ Department of Neurology, the North Hospital, \\ Shenyang, Liaoning 110016, P.R. China
}

Received March 15, 2012; Accepted May 28, 2012

DOI: $10.3892 / \mathrm{mmr} .2012 .937$

\begin{abstract}
It is not clear whether or not the mineralocorticoid receptor (MR) is involved in post-traumatic stress disorder (PTSD). The purpose of this study was to provide novel insights into the mechanism(s) through which the medial prefrontal cortex (mPFC) plays a role in PTSD by investigating MR expression in the $\mathrm{mPFC}$ of rats exposed to single prolonged stress (SPS), which is an established animal model for PTSD. A total of 90 healthy, male Wistar rats were selected for this study and randomly divided into normal control and SPS groups of 1, 7, 14 and 28 days. This study investigated the changes in MR expression in the mPFC of rats after SPS, which revealed pathogenetic mechanisms. The expression of MR in the mPFC was examined by immunofluorescence, western blotting and reverse transcription-polymerase chain reaction (RT-PCR). SPS exposure resulted in a significant change in MR expression in the SPS model groups compared with the normal control group. The MR protein was found to be localized in the cytoplasm and its expression levels were significantly increased in SPS rats, peaking at SPS 7 days, followed by a gradual decrease; however, a positive expression revealed a restoratory increase in the SPS-28 day group. The results suggest that MR plays an important role in the pathology of PTSD.
\end{abstract}

\section{Introduction}

Post-traumatic stress disorder (PTSD) is an anxiety disorder that develops following exposure to a life-threatening traumatic experience and is characterized by symptoms that often endure for years, including continuous re-experience of the traumatic

Correspondence to: Professor Yu-Xiu Shi, Departmant of Histology and Embryology, Basic Medical Sciences College, China Medical University, 92 North 2nd Road, Shenyang, Liaoning 110001, P.R. China

E-mail: shiyuxiu@163.com

Key words: post-traumatic stress disorders, medial prefrontal cortex, mineralocorticoid receptors, single prolonged stress event, avoidance of stimuli associated with the trauma, numbing of general responsiveness and increased arousal (1). The pathophysiology of PTSD has been studied widely in neuroscience (2), however, the mechanism is not fully understood.

It is well known that the dysfunction of the hypothalamopituitary-adrenal (HPA) axis is one of the core neuroendocrine abnormalities correlated with PTSD, characterized by low levels of adrenocorticotropic hormone (ACTH), plasma and urinary cortisol and enhanced suppression of cortisol in response to a low-dose administration of dexamethasone (3-6). Singleprolonged stress (SPS) is a reliable animal model of PTSD based on the time-dependent dysregulation of the HPA axis and has been developed and employed for PTSD research (7-9). A number of studies have shown that the medial prefrontal cortex (mPFC) is important in modulating the responses of the HPA axis to emotional stress $(10,11)$. Thus, the mPFC has been investigated to reveal the mechanism(s) of PTSD.

There are low levels of plasma glucocorticoid (GC) in PTSD (2). Negative feedback on the HPA axis regulated by $\mathrm{GC}$ occurs via the high density of corticosteroid receptors (CsRs) (12). CsRs in the brain can be divided into two types, I and II. Type I has a high affinity for corticosterone and is similar to the human mineralocorticoid receptor (MR). Type II has a low affinity for corticosterone, corresponding to the human glucocorticoid receptor (GR) (13). MR and GR are involved in the physiological regulation of the HPA axis. MR is involved in the negative feedback regulation of the HPA axis at the basic level, which maintains basal activities of the HPA axis and is correlated with the threshold and sensitivity of the central stress response (14).

Little is currently known regarding MR in the brain, particularly in the mPFC, due to the differences between the roles of MR in the brain and other areas. In this study, MR expression in the mPFC of PTSD rats was examined in order to provide experimental evidence for further studies related to the pathogenesis of HPA.

\section{Materials and methods}

Experimental animals. A total of 80 male Wistar rats aged 7 or 8 weeks at the start of the study and weighing approximately 
$180 \mathrm{~g}$ were provided by the Animal Experimental Center, China Medical University (Shenyang, China). Rats were individually housed in an air-conditioned room $\left(22 \pm 1^{\circ} \mathrm{C}\right.$ and $55 \pm 5 \%$ humidity) on a 12 -h light/dark cycle with free access to food and water.

The rats were raised in the laboratory for at least 7 days prior to conducting the experiment. Experiments were carried out in accordance with the National Institute of Health Guide for the care and use of laboratory animals. All efforts were made to reduce the number of animals used and to minimize animal suffering during the experiment.

Model establishment and grouping. Animals were divided randomly into 5 groups $(n=18)$ : i) control; ii) SPS 1 day; iii) SPS 7 days; iv) SPS 14 days; v) SPS 28 days. Control animals remained in their home cages with no handling for 7 days and were sacrificed at the same time as the SPS groups. SPS-rats underwent the SPS procedure on the first day. The SPS protocol was based on a combined plural stress para$\operatorname{digm}(7,15)$ : immobilization (compression with plastic bags) for $2 \mathrm{~h}$, forced swimming for $20 \mathrm{~min}\left(24 \pm 1^{\circ} \mathrm{C}\right)$, rest for $15 \mathrm{~min}$, followed by ether anesthesia (until consciousness was lost). The rats were returned to their home cage and left undisturbed until they were sacrificed for experiments.

Perfusion-based sections. Rats from both the normal control and SPS groups were prepared by left ventricle perfusion and fixation (16) and post-fixed in the same fixative at $4^{\circ} \mathrm{C}$ for $6-10 \mathrm{~h}$. The samples were then immersed in a $20 \%$ sucrose solution in $0.01 \mathrm{M}$ phosphate buffer (PB, pH 7.4) at $4^{\circ} \mathrm{C}$. Samples were fast frozen on liquid nitrogen and sectioned, $12-\mu \mathrm{m}$ coronal sections were prepared for morphological studies.

Immunohistochemical analysis of MR. The sections were treated with $5 \%$ bovine serum albumin (BSA), and $0.3 \%$ Triton X-100 in PBS for $30 \mathrm{~min}$ at room temperature (RT) to block non-specific staining. The sections were then incubated with rabbit polyclonal antibody against MR (Santa Cruz Biotechnology, Inc., Santa Cruz, CA, USA; 1:500) in 2\% BSA-PBS overnight at $4^{\circ} \mathrm{C}$. After being washed with PBS, the sections were incubated with goat anti-rabbit IgG (Boster Biological Technology, Ltd., Wuhan, China; 1:100) for $2 \mathrm{~h}$ and then with streptomycin-avidin-biotin-peroxidase complex (SABC) for $1 \mathrm{~h}$. The sections were washed three times with PBS after each incubation and subsequently incubated with 3,3'-diaminobenzidine (DAB) and $\mathrm{H}_{2} \mathrm{O}_{2}$. To assess non-specific staining, a few sections in every experiment were incubated in buffer without primary antibody.

Five slides were randomly selected from each group. Each slide was randomly selected from five visual fields in the mPFC (x400). The optical density (OD) of positive cells in each field was recorded to evaluate the average. The OD of the MR-immunopositive cells was analyzed using a MetaMorph/ DPIO/BX41 morphology image analysis system.

Western blotting of $M R$. Rats of each group were rapidly decapitated and the brains were removed and immediately placed in a dish standing on crushed ice. The mPFC was then dissected according to the rat brain atlas of Paxinos et al (17) using a stereomicroscope, and snap-frozen in liquid nitrogen.
Table I. MR and $\beta$-actin sequences.

\begin{tabular}{lccc}
\hline Name & $\begin{array}{c}\text { Upstream } \\
\text { primer 5'-3' }\end{array}$ & $\begin{array}{c}\text { Downstream } \\
\text { primer 5'-3' }\end{array}$ & $\begin{array}{c}\text { Product } \\
\text { size (bp) }\end{array}$ \\
\hline MR & agaagatgcatcagtctgcc & gtgatgatctccaccagcat & 380 \\
$\beta$-actin & atcacccacactgtgcccatc & acagagtacttgcgctcagga & 542 \\
\hline
\end{tabular}

$\mathrm{MR}$, mineralocorticoid receptor.

Materials were obtained as above. Samples of normal control and SPS rats were homogenized with a sample buffer containing $200 \mathrm{mM}$ TBS, pH 7.5, 4\% SDS, 20\% glycerol, 10\% 2-mercaptoethanol and were denatured by boiling for $3 \mathrm{~min}$. The protein fraction (30 $\mu \mathrm{g} /$ lane) prepared from each sample was separated by $12 \%$ (w/v) gradient sodium dodecyl sulfate (SDS)-polyacrylamide gel electrophoresis (PAGE) and electroblotted onto a PVDF membrane (Millipore, Bedford, MA, USA) from the gel by a semi-dry blotting apparatus (Bio-Rad Laboratories, Inc., Hercules, CA, USA).

The membrane was blocked with 5\% dried skimmed milk, $0.05 \%$ Tween-20 in TBST at room temperature for $2 \mathrm{~h}$ and incubated with rabbit anti-MR polyclonal antibody $(1: 1,000)$ overnight at $4^{\circ} \mathrm{C}$.

The blots were washed three times with TBST and then incubated with goat anti-rabbit IgG-HRP (Santa Cruz Biotechnology Inc.; 1:5,000) for $2 \mathrm{~h}$ at room temperature and washed with TBST. Following incubation, the PVDF membrane was washed three times with TBST prior to visualization by enhanced chemiluminescence (ECL; Amersham Pharmacia Biotech, Buckinghamshire, UK). To confirm equal protein loading the same blots were reincubated with antibodies specific for $\beta$-actin (Abcam, Cambridge, UK; 1:2,500). $\beta$-actin immunoreactivity was detected using ECL. The OD was analyzed on the Gel Image Analysis System (Tanon 2500R, Shanghai, China). Each MR band was normalized with respect to its corresponding $\beta$-actin band and the values were expressed as an intensity ratio.

Reverse transcription-polymerase chain reaction ( $R T-P C R)$ was used to detect MR. Total mRNA of each group was extracted from the mPFC according to the instructions of the TRIzol kit (Invitrogen, Carlsbad, CA, USA) and $1 \mu \mathrm{g}$ of total RNA was reverse transcribed into cDNA. An RNA PCR kit (AM Ver. 3.0, Takara Bio, Inc., Otsu, Japan) was used to amplify the cDNA. The primers were designed and synthesized by Shenggong Biotech Company (Shanghai, China) according to the serial number from Genbank (Table I). The reaction was started at $94^{\circ} \mathrm{C}$ for $4 \mathrm{~min}$ and amplified to 36 cycles of $45 \mathrm{sec}$ at $94^{\circ} \mathrm{C}, 40 \mathrm{sec}$ at $60^{\circ} \mathrm{C}, 50 \mathrm{sec}$ at $72^{\circ} \mathrm{C}$ and ended with a 10 -min extension at $72^{\circ} \mathrm{C}$. $\beta$-actin mRNA used as an internal control was co-amplified with MR-mRNA. The products were observed after electrophoresis on $1.2 \%$ agarose gel and the OD of each band was analyzed on the Gel Image Analysis System. The level of MR-mRNA was determined by calculating the density ratio of MR mRNA/ $\beta$-actin mRNA.

Statistical analysis. Data were shown as the means \pm standard error. Data among groups were analyzed by one-way analysis 


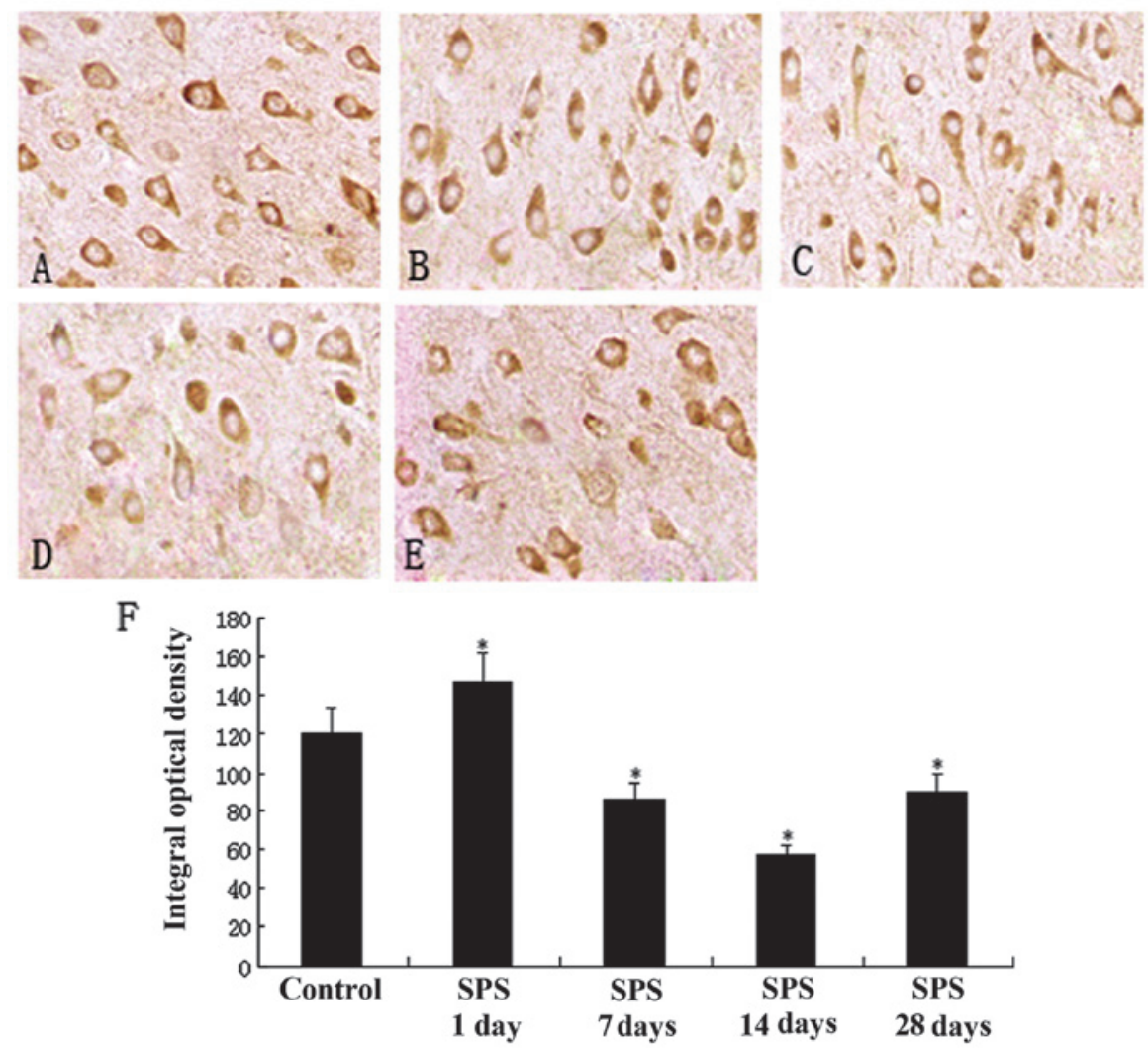

Figure 1. Presentation of MR expression in the mPFC of each group (A-E) magnification, $x 400$. (A) Control group; (B) SPS 1 day; (C) SPS 7 days; (D) SPS 14 days; (E) SPS 28 days; (F) Semi-quantitative analysis results of optical density " $\mathrm{P}<0.05$ vs. control group. MR, mineralocorticoid receptor; SPS, single prolonged stress.

of variance (ANOVA) using SPSS 13.0 software (SPSS, Inc., Chicago, IL, USA). $\mathrm{P}<0.05$ was considered to indicate a statistically significant difference.

\section{Results}

Immunohistochemical analysis results of MR. MR was widely distributed throughout the $\mathrm{MPFC}$ region, primarily in the cytoplasm, and appeared as puffy particles (Fig. 1A-E). Evaluation of MR content by immunohistochemistry indicated a significant change in the SPS model groups compared with the normal control group. The staining results showed that the number of MR-positive cells was less in the normal control group (Fig. 1A) and that of SPS rats was significantly increased and peaked at 7 days after exposure to SPS $(\mathrm{P}<0.01$; Fig. $1 \mathrm{C})$. Positive MR expression gradually decreased in the SPS-14 day group (Fig. 1D). However, in the SPS-28 day group positive expression increased again (Fig. 1E), but remained lower than that of the control group. The analysis results are shown in Fig. $1 \mathrm{~F}(\mathrm{P}<0.05)$

Western blotting analysis of MR. Immunoreactive signals for MR and $\beta$-actin appeared on western blotting at 112 and $42 \mathrm{kDa}$, respectively (data not shown), while the mean value of band densities of the control group was set as 100\%. Data were expressed as normalized OD. Changes of MR expression in the mPFC region between the control and SPS groups are shown in Fig. $2(\mathrm{P}<0.05)$. The MR protein expression of mPFC showed a significant change following SPS stimulation

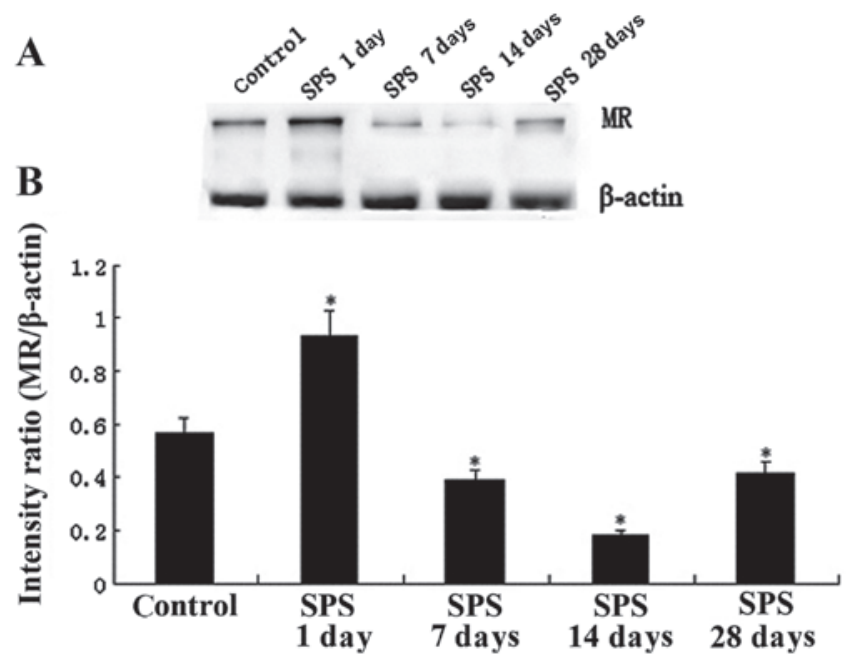

Figure 2. MR expression in mPFC by western blotting. (A) Bands show MR protein levels. (B) Relative quantitative data levels of $\mathrm{MR}$ are shown. ${ }^{*} \mathrm{P}<0.05$ vs. control group. MR, mineralocorticoid receptor; mPFC, medial prefrontal cortex; SPS, single prolonged stress.

and the trend of change was consistent with the results of the immunohistochemistry.

RT-PCR analysis of MR $m R N A$. The levels of MR mRNA were normalized with the $\beta$-actin mRNA level. The results indicated a significant change following SPS stimulation and the trend of 
A
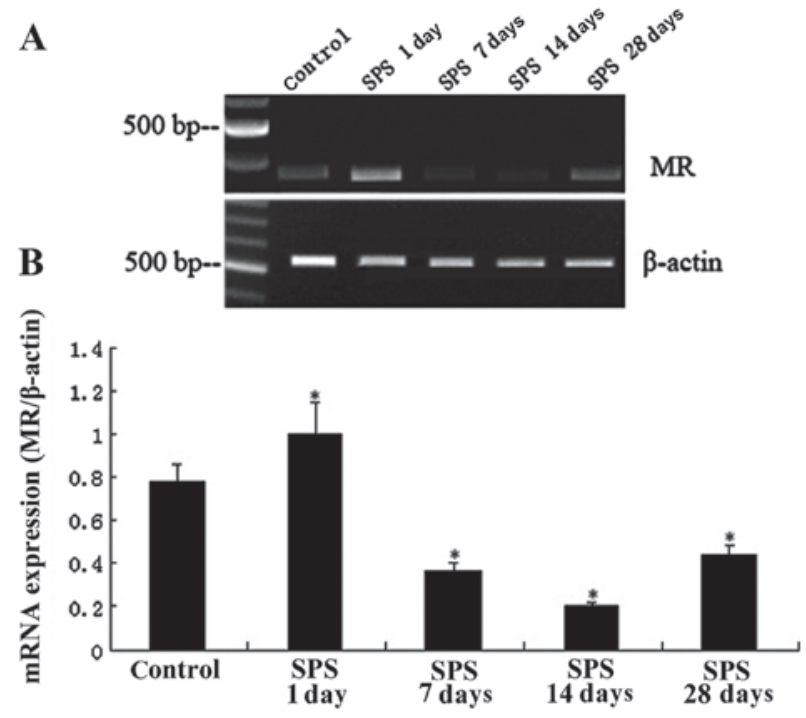

Figure 3. The representative gel pattern of MR, (A) normalized with $\beta$-actin cDNA bands and (B) relative levels of MR mRNA. Lane 0, marker; lane 1, control; lane 2, SPS 1 day; lane 3, SPS 7 days; line 4, SPS 14 days; line 5, SPS 28 days. ${ }^{*} \mathrm{P}<0.05$ as compared with control group. MR, mineralocorticoid receptor; SPS, single prolonged stress.

the change was consistent with the results of the immunohistochemical and western blot analysis $(\mathrm{P}<0.05$; Fig. 3).

\section{Discussion}

PTSD is thought to involve a dysfunction in the response to fear-related stimuli. The four major characteristic symptoms of PTSD are: re-experiencing, avoidance, numbing and hyperarousal (1).

Previous studies have paid little attention to the role of MR in the rat central nervous system compared with that of GR and have mainly investigated an adjusting function of the water-salt balance in epithelial cells. The present opinion is that MR exists in the brain tissue, most substantially in the hippocampus. GR and MR coexist in certain brain regions, including the hippocampus CA1, CA2, CA3 and dentate gyrus sub-region (18). The choice function of MR on mineralocorticoid (aldosterone) in the hippocampus is different from that in epithelial cells. GC combines with the two sets of receptors, GR and MR, at the same time after stimulation. The binding affinity of corticosterone to MR is approximately 10 times higher than that to GR. Low levels of GC combine with MR first, only high levels of GC can combine with GR after MR is saturated. MR is a high-affinity and low-capacity receptor system for GC, while GR is a low-affinity and high-capacity receptor system for GC (19).

The effects of corticosterone are mediated by MR and are also regulated by GR. GR is constantly occupied during the physiological rhythm rising period following GC stress and GC affects the paraventricular nucleus (PVN) through negative feedback. The hippocampus maintains excitability when MR activity dominates and inhibition projects to the PVN through cross-synapses, inhibiting the basic activity of the HPA. Instead, the activation of GR inhibits hippocampal signal output with the increase of GC concentrations, which leads to inhibition of the PVN neurons. The functions of the two types of receptor are interrelated (20). MR defects make corticosterone responses occur more quickly and produce a more GR-mediated effect. The above information shows the importance of MR- and GR-mediated effects in balancing HPA regulation. Therefore, more and more researchers support the balance theory of the receptor-mediated role, which assumes that MR primarily maintains the function of GC and GR is mainly involved in a GC-negative feedback role (21). Since the affinity between MR and GC is higher than that between GR and GC, in the early HPA axis activation or basic level, GC first combines with the MRs of the hippocampus and maintains basal HPA activity, known as proactive feedback. When the GC concentration continues to rise, the high concentration GR continues to combine with GC, inhibiting HPA axis activity via GR-negative feedback, known as reactive feedback (22). GC allows HPA activity to remain at the appropriate level through two types of feedback modes, MR- or GR-mediated, imbalance of which leads to disease. Long-term abnormal corticosteroid levels affect the hippocampal cell response and endanger cell survival (23). Therefore, the hippocampus also functions as a control site of the HPA axis.

One study has shown that MR also exists in the mPFC, with the exception of the hippocampus (20). Selective knockout of MR from the mouse forebrain damages spatial learning ability. By contrast, selective expression of MR in the forebrain reduces anxious behavior of the mouse after stress (20), indicating that MR also exists outside the area of the hippocampus, but its mechanism is not clear. In this experiment MR expression is mainly observed in the cytoplasm of the $\mathrm{mPFC}$, as the transcription role has not yet occurred in the nucleus. MR expression was upregulated significantly after SPS and then decreased 7 days after SPS stimulation, indicating that there was not only a feedback-regulated increase of GR in the hippocampus after stress, but also a change of MR in the mPFC. Expression of MR gradually decreased, but was not restored until 28 days later and differed from that prior to the experiment. This finding indicates that changes of MR in the $\mathrm{mPFC}$ were in accordance with HPA axis regulation, as well as changing with time after SPS stimulation. However, the change of MR in the MPFC may be the result of an HPA axis change, or an MR change leading to an HPA axis change, as the role of MR closely correlates with that of GR, which may play a different role in the same cells. Further studies are needed to confirm the mechanism by which changes in MR occur.

This study revealed the presence of $\mathrm{MPFC}-\mathrm{MR}$ at the protein level using immunohistochemistry and western blotting. There were also quantitative changes in PTSD experimental animals, which all provided an experimental basis for revealing the pathogenetic mechanism of PTSD. Further studies are needed to explore the relevant functional mechanisms and effective interventions in order to find new methods of treatment. However, this experiment is limited to the study of mPFC-MR receptors of PTSD rats, thus, receptor changes of other parts in the brain and its effects on the HPA-axis need further study.

At present, the pathogenesis of PTSD is not yet entirely clear. PTSD may cause a series of biochemical abnormalities and dysfunction of the mPFC, leading to dysfuction of the brain. Thus, more studies should be conducted to determine the pathogenesis of PTSD. 


\section{Acknowledgements}

This research was supported by a grant from the National Natural Science Foundation of China (No. 81171282). The authors would like to thank the anonymous reviewers for their valuable comments on how to improve the quality of the paper.

\section{References}

1. American Psychiatric Association: Diagnostic and Statistical Manual of Mental Disorders, 4th edition. DSM-IV. American Psychiatric Press, Washington, DC, 1994.

2. de Kloet ER: Stress: a neurobiological perspective. Tijdschr Psychiatr 51: 541-550, 2009 (In Dutch).

3. Yehuda R, Halligan SL, Grossman R, et al: The cortisol and glucocorticoid receptor response to low dose dexamethasone administration in aging combat veterans and holocaust survivors with and without posttraumatic stress disorder. Biol Psychiatry 52: 393-403, 2002.

4. Bachmann AW, Sedgley TL, Jackson RV, Gibson JN, Young RM and Torpy DJ: Glucocorticoid receptor polymorphisms and post-traumatic stress disorder. Psychoneuroendocrinology 30 : 297-306, 2005.

5. Yehuda R, Golier JA, Halligan SL, Meaney M and Bierer LM: The ACTH response to dexamethasone in PTSD. Am J Psychiatry 161: 397-1403, 2004.

6. de Kloet CS, Vermetten E, Geuze E, Kavelaars A, Heijnen CJ and Westenberg HG: Assessment of HPA-axis function in posttraumatic stress disorder: pharmacological and non-pharmacological challenge tests, a review. J Psychiatr Res 40: 550-567, 2006.

7. Takahashi T, Morinobu S, Iwamoto Y and Yamawaki S: Effect of paroxetine on enhanced contextual fear induced by single prolonged stress in rats. Psychopharmacology (Berl) 189: 165-173, 2006.

8. Iwamoto Y, Morinobu S, Takahashi T and Yamawaki S: Single prolonged stress increases contextual freezing and the expression of glycine transporter 1 and vesicle-associated membrane protein 2 mRNA in the hippocampus of rats. Prog Neuropsychopharmacol Biol Psychiatry 31: 642-651, 2007.

9. Khan S and Liberzon I: Topiramate attenuates exaggerated acoustic startle in an animal model of PTSD.Psychopharmacology (Berl) 172: 225-229, 2004.

10. Figueiredo HF, Bruestle A, Bodie B, Dolgas CM and Herman JP: The medial prefrontal cortex differentially regulates stressinduced c-fos expression in the forebrain depending on type of stressor. Eur J Neurosci 18: 2357-2364, 2003.
11. Radley JJ, Arias CM and Sawchenko PE: Regional differentiation of the medial prefrontal cortex in regulating adaptive responses to acute emotional stress. J Neurosci 26: 12967-12976, 2006.

12. Nagalski A and Kiersztan A: Physiology and molecular mechanism of glucocorticoid action. Postepy Hig Med Dosw 18: 133-145, 2010 (In Polish)

13. Kessler RC: Posttraumatic stress disorder: the burden to the individual and to society. J Clin Psychiatry 61: 4-12, 2000.

14. Rogalska J: Mineralocorticoid and glucocorticoid receptors in hippocampus: their impact on neurons survival and behavioral impairment after neonatal brain injury. Vitam Horm 3: 391-419, 2010.

15. Kohda K, Harada K, Kato K, Hoshino A, Motohashi J, Yamaji T, Morinobu S, Matsuoka N and Kato N: Glucocorticoid receptor activation is involved in producing abnormal phenotypes of single-prolonged stress rats: a putative post-traumatic stress disorder model. Neuroscience 148: 22-33, 2007.

16. Liu HY: Technical operations and its common problems of perfusion fixation in mice. Qiqihaer Yixueyuan Xuebao 27: 1341, 2006.

17. Paxinos G, Watson CR and Emson PC: AChE-stained horizontal sections of the rat brain in stereotaxic coordinates. J Neurosci Methods 3: 129-149, 1980.

18. Zhe D, Fang H and Yuxiu S: Expressions of hippocampal mineralocorticoid receptor (MR) and glucocorticoid receptor (GR) in the single-prolonged stress-rats. Acta Histochem Cytochem 41: 89-95, 2008.

19. De Kloet ER, Vreugdenhil E, Oitzl MS and Joels M: Brain corticosteroid receptor balance in health and disease. Endocr Rev 19: 269-330, 1998 .

20. Kolber BJ, Wieczorek L and Muglia LJ: Hypothalamic-pituitaryadrenal axis dysregulation and behavioral analysis of mouse mutants with altered glucocorticoid or mineralocorticoid receptor function. Stress 11: 321-338, 2008.

21. Sousa N, Cerqueira JJ and Almeida OF: Corticosteroid receptors and neuroplasticity. Brain Res Rev 57: 561-570, 2008.

22. Nishi M and Kawata M: Dynamics of glucocorticoid receptor and mineralocorticoid receptor: implications from live cell imaging studies. Neuroendocrinology 85: 186-192, 2007.

23. Kellner M, Baker DG, Yassouridis A, Bettinger S, Otte C, Naber D and Wiedemann K: Mineralocorticoid receptor function in patients with posttraumatic stress disorder. Am J Psychiatry 159: 1938-1940, 2002. 